\title{
Anti-toxoplasmosis Effects of Oleuropein Isolated from Fraxinus rhychophylla
}

\author{
Jing-Hua JIANG, ${ }^{a, \#}$ Chun-Mei JIN, ${ }^{a, \#}$ Youn-Chul KIM,${ }^{b}$ Hun-Soo KIM,${ }^{c}$ Won-Cheol PARK, ${ }^{d}$ and \\ Hyun PARK ${ }^{*}, a$ \\ ${ }^{a}$ Department of Infection Biology, Zoonosis Research Center, Wonkwang University School of Medicine; ${ }^{c}$ Department of \\ Pathology, Wonkwang University School of Medicine; ' Department of Surgery, Wonkwang University School of Medicine; \\ 344-2 Shinyong-dong, Iksan, Chonbuk 570-749, Korea: and ${ }^{b}$ College of Pharmacy, Wonkwang University; 344-2 \\ Shinyong-dong, Iksan, Chonbuk 570-749, Korea. Received May 31, 2008; accepted September 16, 2008
}

Toxoplasmosis is a parasitic disease caused by Toxoplasma gondii, with very few therapeutic treatment options. Typically, the choices for treatment are pyrimethamine and sulfadiazine, however their utility is limited because of drug toxicity and serious side effects. For these reasons, new drugs with lower toxicity are urgently needed. In this study, the compound oleuropein isolated from Fraxinus rhynchophylla showed anti-T. gondii effects in vitro and in vivo. In Madin-Darby bovine kidney cells, the selectivity of oleuropein was 8.9, which was higher than sulfadiazine and pyrimethamine (3.8 and 2.5, respectively). In infected mice, the inhibition ratio of $T$. gondii in the peritoneal cavity was $55.4 \%$ compared to the negative control group after treatment with $300 \mathrm{mg} / \mathrm{kg}$ oleuropein. In addition, inhibitory effects on granuloma, apoptosis, necrosis and cyst-formation were shown in sections of spleen and liver. Oleuropein is therefore a potentially useful anti-T. gondii candidate for clinical application.

Key words oleuropein; Toxoplasma gondii; Fraxinus rhynchophylla; toxoplasmosis

Toxoplasma gondii, the cause of toxoplasmosis, is a ubiquitous intracellular parasite. ${ }^{1-3)}$ T. gondii infections are widely prevalent in humans and other animals. ${ }^{4)}$ During its complex life cycle, bradyzoites are less susceptible to chemotherapy, and reactivation of bradyzoites to tachyzoites is considered the cause of fatal toxoplasmosis in many AIDS patients. After infection with any infective stage, tachyzoites multiply in a variety of cells and eventually form cysts in several tissues. ${ }^{4)}$ Arendt et al. $^{5)}$ reported that acute infection or secondary reactivation of toxoplasmosis could lead to psychiatric complications such as disorientation, anxiety, depression and even psychoses upon development of immunosuppression.

Effective drugs used to treat toxoplasmosis are sulfonamide and pyrimethamine, either alone or in synergistic combination. Continuous drug therapy is essential for patients to ensure prevention of severe complications. However, adverse side effects to drugs (e.g. sulfadiazine) such as bone marrow suppression, hypersensitivity and fever are frequently seen. ${ }^{6}$ While other drugs (e.g. clindamycin, atovaquone, daspone, trimethoprim, pentamidine and azithromycin) have anti- $T$. gondii activities and have been used in the clinic, relapse is often seen because of common side-effects. ${ }^{7}$ Herbal extracts are thought to contain the desired properties of potency and low toxicity and show promise for the identification of new chemotherapeutic agents. It had been reported that many herbal extracts recorded in "DONGYIBOGAM" and the dictionary of traditional Chinese medicine contain a variety of chemotherapeutic compounds that have a range of biological activities including anti-fever or anti-inflammatory effects. It was recorded that Fraxinus possessed anti-diarrhea, antibacterial, anti-inflammatory and analgesic properties. Fraxinus rhynchophylla was used for eye disease, eclampsia, dysentery, asthma, bronchitis and leucorrhea. Kim et al. ${ }^{8)}$ found that $F$. rhynchophylla could furnish two inducible nitric oxide synthase (iNOS) inhibitory compounds preventing nitric oxide (NO) synthesis. Moreover, oleuropein (Fig. 1) and its metabolites (chemical compounds found in F. rhynchophylla) have shown powerful antioxidant activities, anti-ischemic and hypolipidemic effects both in vivo and in vitro. ${ }^{9)}$ However, there were no studies concerning the anti- $T$. gondii activities of oleuropein. Therefore, in our studies, a compound oleuropein isolated from $F$. rhynchophylla was investigated for its anti-T. gondii effects in vitro and in vivo.

\section{MATERIALS AND METHODS}

Materials Oleuropein isolated from $F$. rhynchophylla was supplied from Dr. Youn-Chul Kim. Sulfadiazine sodium salt and pyrimethamine were purchased from the Sigma Chemical Co. (St. Louis, MO, U.S.A.). The CellTiter $96^{\circledR}$ $\mathrm{AQ}_{\text {ueous }}$ One Solution Cell Proliferation Assay kit was purchased from the Promega Corporation (Woods Hollow Road, Madison, U.S.A.). All sera, antibiotics and RPMI 1640 for cell culture were obtained from Invitrogen (Grand Island, N.Y. 14072, U.S.A.). All other chemicals were of reagent grade.

Cell Culture and $T$. gondii Maintainence MadinDarby bovine kidney cells (MDBK, CCL-22, ATCC) were cultured in RPMI medium 1640 supplemented with $10 \%$ heat-inactivated fetal bovine serum, 100 units $/ \mathrm{ml}$ penicillin and $100 \mu \mathrm{l} / \mathrm{ml}$ streptomycin with $5 \% \mathrm{CO}_{2}$ at $37^{\circ} \mathrm{C}$. T. gondii

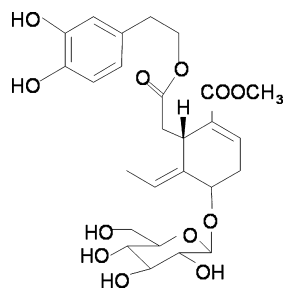

Fig. 1. The Chemical Structure of Oleuropein 
(RH strain) was maintained in KP100 CD-1 female mice (ORIENT BIO CO., LRD, South Korea) and tachyzoites were obtained from peritoneal fluids.

Cell Proliferation Assay The MTS [3-(4,5-dimethylthiazol-zyl)-5-(3-carboxymethoxyphenyl)-2-(4-sulfophenyl)$2 \mathrm{H}$-tetrazoliuzolium, inner salt] assay was used to characterize cellular proliferation using CellTiter $96^{\circledR} \mathrm{AQ}_{\text {ueous }}$ One Solution Cell Proliferation Assay kit. ${ }^{10,11)}$ This assay measures dehydrogenase enzymes in metabolically active cells. ${ }^{12)}$ After MDBK cells were infected with $T$. gondii for $24 \mathrm{~h}$ in 96-well microplates, they were treated with drugs for $24 \mathrm{~h}$. Next, MTS solution $(20 \mu \mathrm{l})$ was added directly into the culture wells, and the plates were incubated for $1.5 \mathrm{~h}$ at $37^{\circ} \mathrm{C}$ followed by a recording of the absorbance at $490 \mathrm{~nm}$ using a microplate reader. Cell viability was expressed as a percent of the control value.

Animal Treatment and Tissue Stain KP100 CD-1 female mice ( 5 weeks, 6 mice/group) were injected with $1 \times 10^{5}$ tachyzoites in the abdominal cavity. Mice were separated into 5 groups: i) normal group (no T. gondii), ii) negative control group (T. gondii and water), iii) positive control group 1 ( $T$. gondii and pyrimethamine), iv) positive control group 2 ( $T$. gondii and sulfadiazine), and v) drug group ( $T$. gondii and oleuropein). After infection for $2 \mathrm{~h}$, the negative control group, two positive control groups and oleuropein group were treated orally with $300 \mu \mathrm{l}$ water, $12.5 \mathrm{mg} / \mathrm{kg}$ pyrimethamine, $50 \mathrm{mg} / \mathrm{kg}$ sulfadiazine, $300 \mathrm{mg} / \mathrm{kg}$ oleuropein, respectively, once a day for $4 \mathrm{~d}$. Animals were sacrificed 4-d post infection. Ascites were drawn out to determine the level of tachyzoites proliferation. ${ }^{13)}$ Spleens and livers were fixed flat in $10 \%$ buffered formalin for $24 \mathrm{~h}$ and sections were stained with hematoxylin and eosin (H\&E) for pathological inspection.

Statistical Analysis Statistical analysis was performed using the Student's $t$-test and nonparametric correlation analysis (Mann-Whitney $U$ test) with SPSS 11.5.1 software for windows. Differences were considered to be significant at a level of $p<0.05$.

\section{RESULTS AND DISCUSSION}

T. gondii can infect nearly all kinds of vertebrates and is clinically significant due to its ability to cause toxoplasmosis. Pathogenesis shows the interconversion of the parasite between the tachyzoite stage (acute infection) and the bradyzoite stage (chronic infection). In this study, oleuropein, a compound isolated from $F$. rhynchophylla, was investigated for its anti-tachyzoite proliferation effect in mice. It has already been reported that oleuropein has anti-inflammatory and anti-oxidative properties on bone loss induced by talc granulomatosis in oestrogen-deficient rats. ${ }^{14)}$ However, this compound has not been characterized for potential anti-T. gondii effects.

In our study, the inhibition of infection ratio of host cells was determined using the MTS assay and morphological assays (data not shown). The results of the MTS assay were close to those of the morphological assay and had a high $R$ value $(R=0.9841)$. Therefore, the EC50 values were measured using the MTS assay. The effect of oleuropein on cell proliferation after treatment with T. gondii is shown in Fig. 2. The viability was reduced in a dose-dependent manner. The

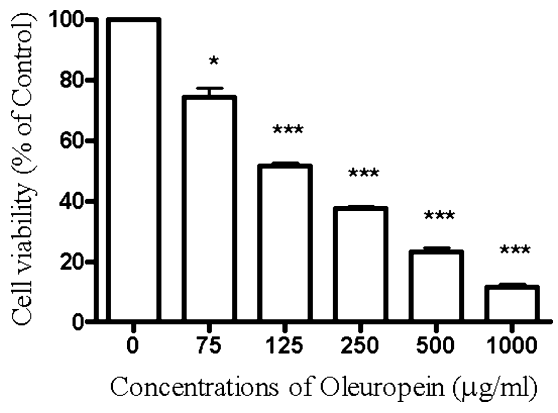

Fig. 2. The Effect of Oleuropein on Cell Proliferation Was Shown after Treatment with $T$. gondii by MTS Assay on MDBK Cells

The data were presented as means \pm S.D. and the experiments were performed in triplicate. Statistical analysis was performed using the Student's $t$-test. $* p<0.05$ and $* * * p<0.001$ were considered significant with control level, respectively.

Table 1. Selectivity of Oleuropein in MDBK Cells

\begin{tabular}{lccc}
\hline \hline & $\begin{array}{c}\text { EC50 in MDBK cells } \\
(\mu \mathrm{g} / \mathrm{ml})\end{array}$ & $\begin{array}{c}\text { EC50 in T. gondii } \\
(\mu \mathrm{g} / \mathrm{ml})\end{array}$ & Selectivity \\
\hline Oleuropein & 1233 & 139 & 8.9 \\
Sulfadiazine & 2300 & 600 & 3.8 \\
Pyrimethamine & 338 & 135 & 2.5 \\
\hline
\end{tabular}

selectivity was calculated by the following formula: selectivity $=$ MDBK cell EC50/T. gondii EC50. These data reflected the efficacy of a compound against $T$. gondii and toxicity for host cells. The selectivity of oleuropein, sulfadiazine and pyrimethamine were measured in MDBK cells first and determined to be $8.9,3.8$ and 2.5 , respectively. Oleuropein has a higher selectivity than sulfadiazine and pyrimethamine, and showed good efficacy as an anti-T. gondii compound in vitro (Table 1).

During natural infections, T. gondii initially crosses the intestinal epithelium, disseminates into deep tissues and traverses. In our study, mice were sacrificed 4-d post infection and ascites were used to determine tachyziotes proliferation. The dose-dependent effects of oleuropein (orally 42, 125, $300 \mathrm{mg} / \mathrm{kg}$ ) in mice were examined first. Lower concentrations of 42 and $125 \mathrm{mg} / \mathrm{kg}$ oleuropein did not show an anti-T. gondii effect in the peritoneal cavity (data not shown) but the $300 \mathrm{mg} / \mathrm{kg}$ dose did. Based on a study by Choi et al., $\left.{ }^{15}\right)$ $12.5 \mathrm{mg} / \mathrm{kg}$ pyrimethamine and $50 \mathrm{mg} / \mathrm{kg}$ sulfadiazine were used for this study. It has been reported that oleuropein is a non-toxic secoiridoid derived from the olive tree with a potent anti-cancer effect in vivo. ${ }^{16)}$ Due to this low toxicity, a high concentration $(300 \mathrm{mg} / \mathrm{kg})$ was used in our in vivo experiment, even though it was higher than concentrations of pyrimethamine and sulfadiazine. Numbers of tachyzoites in the peritoneal cavity of the negative group, pyrimethaminetreated positive group, sulfadiazine-treated positive group and oleuropein-treated group were 257.5, 114.6, 22.1 and $114.8\left(\times 10^{5}\right)$, respectively (Fig. 3). The inhibition ratio of $T$. gondii in the peritoneal cavity was $55.4 \%$ compared to the negative control group after treatment with $300 \mathrm{mg} / \mathrm{kg}$ oleuropein. It was shown to be statistically significant compared to the negative control group. Although its inhibition ratio was not better than sulfadiazine treatment $(85.3 \%)$, its level nearly reached the inhibition level of pyrimethamine $(55.5 \%)$. These results suggest that oleuropein has an anti-T. 
gondii effect in mice peritoneal cavities like pyrimethamine.

In addition, in order to compare the anti- $T$. gondii effect of oleuropein with pyrimethamine or sulfadiazine-treated positive group, sections of the liver and spleen from infected mice were analyzed pathologically (Fig. 4, Table 2). The tissue sections from infected mice were stained by H\&E. In liver tissues from the negative control group, a moderate level of granuloma was observed; amounts of granuloma were 4/10 HPF (High Power Field), and they had 1 cyst/50 HPF. In the sulfadiazine and pyrimethamine groups, granuloma levels were mild: 1/10 HPF, and they had 0 cysts/50 HPF. In the oleuropein group, granuloma levels were mild: 2/10 HPF, and they had 0 cysts/50 HPF. In spleen tissues, levels of apoptosis and necrosis of the oleuropein group were mild and negative, respectively. This was better than the negative group, which showed moderate and positive levels, similar to the pyrimethamine group, which showed mild and negative levels, however not better than the sulfadiazine group, which showed all negative levels. Spleens from the negative group had 11 cysts/50 HPF, but the oleuropein group and positive group had only 1 cyst (/50 HPF) (Table 2 ). These results suggested that oleuropein had an inhibitory effect on cyst development in not only the liver but also in the spleen like sulfadiazine and pyrimethamine, and had good anti-granuloma, anti-apoptosis, anti-necrosis effects in T. gondii-infected mice. Compared with the above results in the abdominal cavity, the anti-T. gondii effect of sulfadiazine, pyrimethamine and oleuropein were different. In our in vivo model, sulfadiazine showed a more potent inhibitory effect on tachyzoits than pyrimethamine in the abdominal cavity of

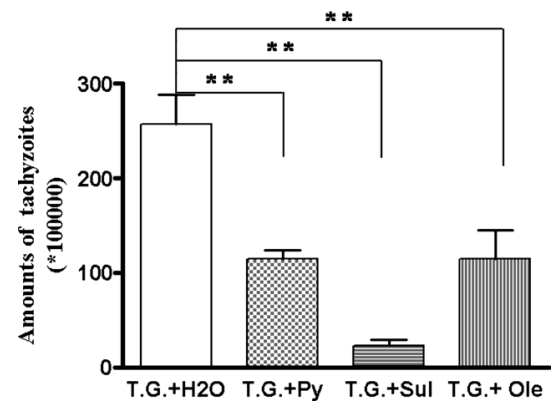

Fig. 3. Amount of Tachyzoites in Mouse Peritoneal Cavity

After mice were infected with $T$. gondii $\left(10^{5} /\right.$ mouse) for $2 \mathrm{~h}$, the negative control group, positive contrast groups and oleuropein group were treated orally by gavage once a day for $4 \mathrm{~d}$ with $300 \mu \mathrm{l}$ water, $12.5 \mathrm{mg} / \mathrm{kg}$ pyrimethamine, $50 \mathrm{mg} / \mathrm{kg}$ sulfadiazine or $300 \mathrm{mg} / \mathrm{kg}$ oleuropein, respectively. All mice were sacrificed $4-\mathrm{d}$ post infection and tachyzoites were harvested from mice peritoneal cavities (6 mice/group). $* * p<0.01$ was considered significant compared to the control level. T.G.: T. gondii; Py: pyrimethamine; Sul: sulfadiazine Ole: oleuropein.

Table 2. Histological Analysis in T. gondii-Inoculated Mice

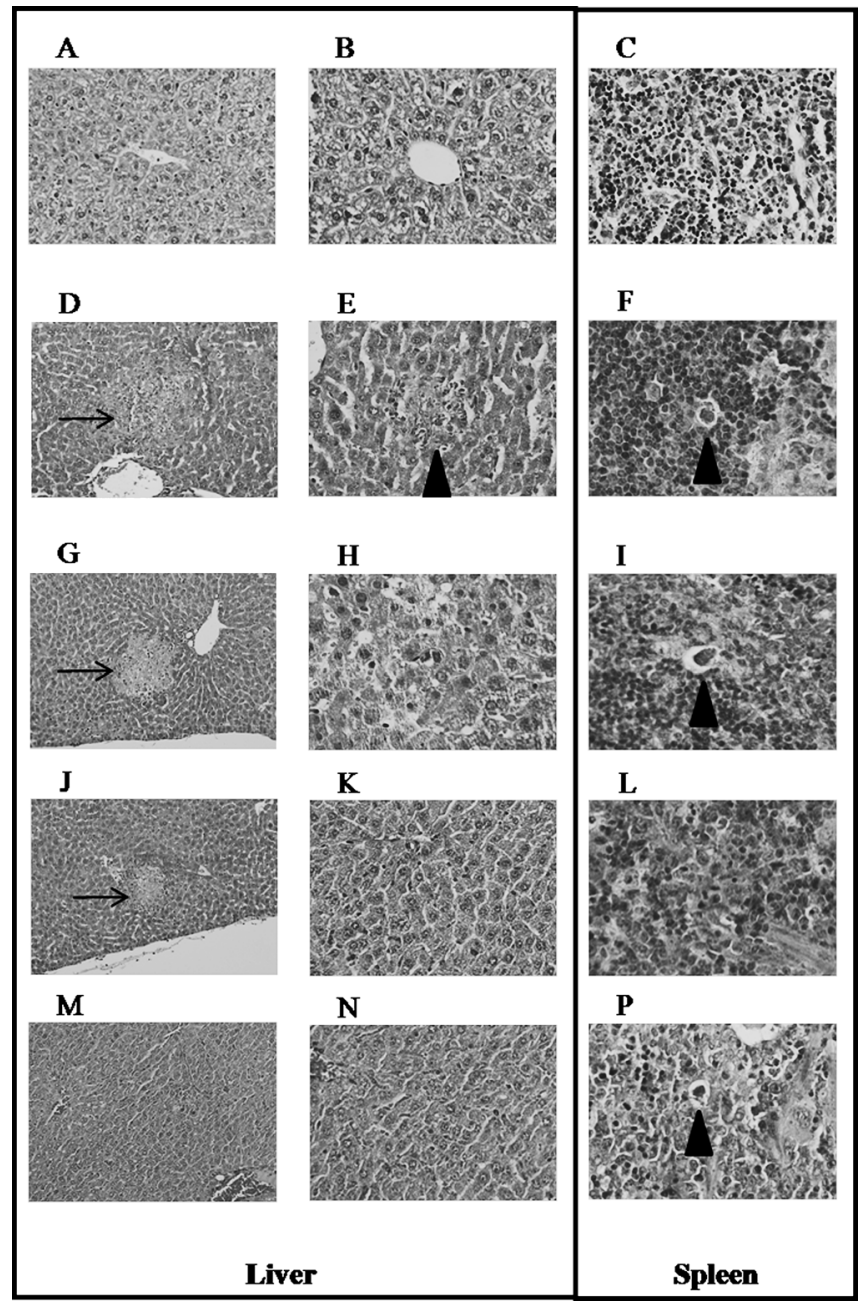

Fig. 4. Histopathological Photos of Mice Livers and Spleens $4 \mathrm{~d}$ after Treatment with Chemicals (H\&E Stain)

A, B and C: Normal liver parenchyma without granulomas or cysts in the control group. Splenic tissue with normal red and white pulp without apoptosis or necrosis in the control group; D, E and F: T. gondii-infected group shows moderate degree of granulomatous inflammation with a cyst (1/50 HPF) in the liver (D and E). Moderate degree of apoptosis with necrosis and numerous cysts infesting the spleen (11/50 HPF) (F); G, $\mathrm{H}$ and I: Pyrimethamine-treated group shows a significant decrease in the degree of granulomatous inflammation, in the liver $(\mathrm{G}$ and $\mathrm{H})$ and apoptosis and necrosis in the spleen, with a few cysts infesting the spleen (1/50 HPF) (I); J, K and L: The sulfadiazine-treated group also shows a significant decrease in the degree of granulomatous inflammation, in the liver ( $\mathrm{J}$ and $\mathrm{K}$ ). The spleen shows negative apoptosis and necrosis (L). There are no cysts infecting the liver or the spleen; M, N and P: The oleuropeintreated group shows a decrease in granulomatous inflammation in the liver, decrease in apoptosis and necrosis with occasional cysts in the spleen, but to a lesser extent than the other pyrimethamine- and sulfadiazine-treated group. A, D, G, J and $M$ are $\times 200$; others are $\times 400$. Arrow indicates granulomatous and triangle indicates cyst. HPF: High Power Field.

\begin{tabular}{|c|c|c|c|c|c|}
\hline \multirow{2}{*}{ Group } & \multicolumn{2}{|c|}{ Liver } & \multicolumn{3}{|c|}{ Spleen } \\
\hline & $\begin{array}{l}\text { Amount of Gr. } \\
\text { (/10HPF) }\end{array}$ & $\begin{array}{l}\text { Amount of cyst } \\
\text { (/50HPF })\end{array}$ & Apoptosis & Necrosis & $\begin{array}{c}\text { Amount of cyst } \\
\text { (/50HPF) }\end{array}$ \\
\hline Normal & Negative/0 & 0 & Negative & Negative & 0 \\
\hline T.G.+D.W. & Moderate/4 & 1 & Moderate & Positive & 11 \\
\hline T.G. + Py & Mild/1 & 0 & Mild & Negative & 1 \\
\hline T.G.+Sul & Mild/1 & 0 & Negative & Negative & 1 \\
\hline T.G. + Ole & Mild/2 & 0 & Mild & Negative & 1 \\
\hline
\end{tabular}

Degree of granuloma: negative: none/10HPF; mild: $1-3 / 10 \mathrm{HPF}$; moderate: $4-6 / 10 \mathrm{HPF}$; severe: $>6 / 10 \mathrm{HPF}$; HPF: high power field. Degree of apoptosis and necrosis: negative: none; mild: $1-5 \%$; moderate: $5-10 \%$; positive: $>10 \%$. Gr.: granuloma; T.G.: T. gondii; D.W.: distilled water; Py: pyrimethamine; Sul: sulfadiazine; Ole: oleuropein. 
mice, even though their inhibitory effects in tissues were all good. Oleuropein was very similar to pyrimethamine.

In summary, the oleuropein compound isolated from $F$. rhynchophylla showed anti-toxoplasmosis effects in $T$. gondii-infected mice. So, oleuropein is a potentially useful anti- $T$. gondii candidate. We plan to perform additional studies to characterize the anti- $T$. gondii effects of these oleuropein derivative compounds. Moreover, we plan to detail the pharmacology indexes, such as long term-effects, side effects, acute toxicity and chronic toxicity test as we progress.

Acknowledgements This work was supported by the Korea Foundation for International Cooperation of Science $\&$ Technology (KICOS) through a grant provided by the Korean Ministry of Science \& Technology (MOST) in No. 2007-00208 and by a grant No. RTI05-03-02 from the Regional Technology Innovation Program of the Ministry of Commerce, Industry and Energy (MOCIE), Korea.

\section{REFERENCES}

1) Dukes C., Luft B., Durack D., "Toxoplasmosis, in Infections of the Central Nervous System," ed. by Scheld W. M., Whitley R. J., Durack D. T., Lippincott-Raven, Philadelphia, 1997, pp. 785-806.

2) Remington J. S., McLeod R., Thulliez P., Desmonts G., "Toxoplasmosis in Infectious Diseases of the Fetus and Newborn Infant," 5th ed., ed. by Remington J. S., Klein J. O., WB Saunders, Philadelphia, 2001, pp. $205-346$.

3) Alan S. B., Catherine A. S., Charles P. Q., Liyan L., Vicki P. B., Ezra S. S., Am. J. Psychiatry, 162, 767-773 (2005).

4) Dubey J. P., Beattie C. P., "Toxoplasmosis of Animals and Man," CRC Press, Boca Raton, Florida, 1988.

5) Arendt G., Von Giesen H. J., Hefter H., Neuen-Jacob E., Roick H., Jablonowski H., Acta Neurol. Scand., 100, 178-184 (1999).

6) McFadden D. C., Seeber F., Boothroyd J. C., Antimicrob. Agents Chemother., 41, 1849-1853 (1997).

7) Georgiev V. S., Drugs, 48, 179-188 (1994).

8) Kim N. Y., Pae H. O., Ko Y. S., Yoo J. C., Choi B. M., Jun C. D., Chung H. T., Inagaki M., Higuchi R., Kim Y. C., Planta Med., 65, 656-658 (1999).

9) Andreadou I., Iliodromitis E. K., Mikros E., Constantinou M., Agalias A., Magiatis P., Skaltsounis A. L., Kamber E., Tsantili-Kakoulidou A., Kremastinos D. T., J. Nutr., 136, 2213-2219 (2006).

10) Cory A. H., Owen J. C., Barltrop J. A., Cory J. G., Cancer Commun., 3, 207-212 (1991).

11) Riss T. L., Moravec R. A., Mol. Biol. Cell, 3, 184a (1992).

12) Berridge M. V., Tan A. S., Arch. Biochem. Biophys., 303, 474- 482 (1993).

13) Murakami N., Sugimoto M., Kawanishi M., Tamura S., Kim H. S., Begum K., Wataya Y., Kobayashi M., J. Med. Chem., 46, 638-641 (2003).

14) Puel C., Mathey J., Agalias A., Kati-Coulibaly S., Mardon J., Obled C., Davicco M. J., Lebecque P., Horcajada M. N., Skaltsounis A. L., Coxam V., Clin. Nutr., 25, 859-868 (2006).

15) Choi J. S., Chung P. R., Soh C. T., Yonsei J. Med. Sci., 18, 340-354 (1985).

16) Hamdi H. K., Castellon R., Biochem. Biophys. Res. Commun., 334, $769-778$ (2005). 